\title{
Loss of Neurons from Laminas I-III of the Spinal Dorsal Horn Is Not Required for Development of Tactile Allodynia in the Spared Nerve Injury Model of Neuropathic Pain
}

\author{
Erika Polgár, David I. Hughes, Ahmad Z. Arham, and Andrew J. Todd \\ Spinal Cord Group, Institute of Biomedical and Life Sciences, University of Glasgow, Glasgow G12 8QQ, United Kingdom
}

\begin{abstract}
It has been proposed that death of inhibitory interneurons in the dorsal horn contributes to the neuropathic pain that follows partial nerve injury. In this study, we have used two approaches to test whether there is neuronal death in the dorsal horn in the spared nerve injury (SNI) model. We performed a stereological analysis of the packing density of neurons in laminas I-III 4 weeks after operation and found no reduction on the ipsilateral side compared with that seen on the contralateral side or in sham-operated or naive rats. In addition, we used two markers of apoptosis, terminal deoxynucleotidyl transferase-mediated biotinylated UTP nick end labeling (TUNEL) staining and immunocytochemical detection of cleaved (activated) caspase-3. Neither of these methods demonstrated apoptotic neurons in the dorsal spinal cord 1 week after operation. Although TUNEL-positive cells were present throughout the gray and white matter at this stage, they were virtually all labeled with antibody against ionized calcium-binding adapter molecule 1, a marker for microglia. All animals that underwent SNI showed clear signs of tactile allodynia affecting the ipsilateral hindpaw. These results suggest that a significant loss of neurons from the dorsal horn is not necessary for the development of tactile allodynia in the SNI model.
\end{abstract}

Key words: disinhibition; TUNEL; caspase; apoptosis; microglia; cell death

\section{Introduction}

Injury to peripheral nerves often leads to neuropathic pain, which is thought to result from both abnormal excitability of damaged primary afferents and pathological changes within the CNS. A relatively common symptom is tactile allodynia, in which innocuous mechanical stimuli are perceived as painful. This can be mediated by low-threshold mechanoreceptive $\mathrm{A} \beta$ afferents (Campbell et al., 1988; Koltzenburg et al., 1994), and because stimulation of these does not normally result in pain, it is assumed that a central mechanism must be involved (Woolf, 1997).

One of the central changes that is thought to contribute to neuropathic pain is excitotoxic death of inhibitory interneurons within laminas I-III of the spinal dorsal horn (Zimmerman, 2001; Moore et al., 2002). Evidence for this hypothesis includes the following observations: (1) loss of GABAergic or glycinergic inhibition within the dorsal horn leads to allodynia (Yaksh, 1989) and a lowered threshold for withdrawal reflexes (Sivilotti and Woolf, 1994); (2) there is a reduction in the level of GABA (Castro-Lopes et al., 1993; Ibuki et al., 1997; Eaton et al., 1998) and its synthesizing enzyme glutamate decarboxylase (Moore et al., 2002) in the ipsilateral dorsal horn after nerve injury; (3) "dark neurons" (Sugimoto et al., 1990) and apoptotic cells la-

\footnotetext{
Received April 15, 2005; revised June 1, 2005; accepted June 2, 2005.

We thank R. Kerr, C. Watt, and M. McGill for expert technical assistance, Prof. D. J. Maxwell and Dr. M. Webb for helpful discussion, and the Wellcome Trust for financial support.

Correspondence should be addressed to Dr. A. J. Todd, Spinal Cord Group, West Medical Building, University of

Glasgow, University Avenue, Glasgow G12 8QQ, UK. E-mail: a.todd@bio.gla.ac.uk.

D0I:10.1523/JNEUROSCI.1490-05.2005

Copyright $\odot 2005$ Society for Neuroscience $\quad$ 0270-6474/05/256658-09\$15.00/0
}

beled with the terminal deoxynucleotidyl transferase-mediated biotinylated UTP nick end labeling (TUNEL) method (Kawamura et al., 1997; Azkue et al., 1998; Whiteside and Munglani, 2001; Moore et al., 2002; de Novellis et al., 2004) have been detected in the dorsal horn after various types of nerve injury; (4) a substantial reduction of primary afferent-evoked IPSCs in lamina II neurons has been reported in neuropathic models (but not after sciatic nerve transection) and was thought to result from a presynaptic mechanism involving reduction of GABA release (Moore et al., 2002). Although most studies of apoptosis in the dorsal horn after nerve injury have not identified the cell types involved, Moore et al. (2002) reported that in the spared nerve injury (SNI) model (Decosterd and Woolf, 2000), $\sim 10 \%$ of TUNEL-positive cells were neuronal.

However, despite the evidence that neuronal death contributes to neuropathic pain, Polgár et al. $(2003,2004)$ reported that there was neither a loss of neurons nor a reduction in the proportion of neurons that were GABA immunoreactive in laminas I-III in the chronic constriction injury (CCI) model of neuropathic pain (Bennett and Xie, 1988). In the present study, we tested the hypothesis that there is neuronal loss in the SNI model (in which there is prominent, long-lasting tactile allodynia) by performing a stereological analysis of neuronal packing density in laminas I-III in rats that had undergone SNI 4 weeks previously. We also used two approaches to look for evidence of dying neurons 1 week after SNI: TUNEL staining and detection of cleaved (activated) caspase-3, an "executioner" caspase that is thought to be necessary for neuronal apoptosis (Troy and Salvesen, 2002). This time point was chosen because it has been reported 
that apoptosis in the dorsal horn is maximal $\sim 1$ week after nerve injury (Whiteside and Munglani, 2001).

\section{Materials and Methods}

All experiments were approved by the Ethical Review Process Applications Panel of the University of Glasgow and were performed in accordance with the UK Animals (Scientific Procedures) Act (1986).

Operative procedures and behavioral testing. Operations were performed on 18 adult male Sprague Dawley rats; 12 underwent SNI (Decosterd and Woolf, 2000), and six had a sham operation. For the SNI, rats were anesthetized with halothane, and an incision through skin and muscle was made to expose the left sciatic nerve at the level of its trifurcation. The common peroneal and tibial branches of the sciatic nerve were ligated tightly with 4.0 silk and transected just distal to the ligation. A 2-4 $\mathrm{mm}$ length of each nerve was removed, and the wound was sutured in two layers. Care was taken to avoid damaging the intact sural nerve. Sham operations were also performed under halothane anesthesia and involved exposure of the left sciatic nerve without manipulation. Six of the SNI rats $(270-315 \mathrm{~g})$ and all sham-operated animals (275-310 g) survived for $28 \mathrm{~d}$ after surgery, whereas the remaining six SNI rats (260-310 g) survived for $7 \mathrm{~d}$ after operation.

Behavioral testing to detect signs of tactile allodynia was performed on all operated animals on several occasions: 6, 3, and $1 \mathrm{~d}$ before and 1, 4, and $7 \mathrm{~d}$ after the operation. Those animals that survived $28 \mathrm{~d}$ were also tested on postoperative days 14,21 , and 28 . The animals were placed in a Perspex cage with a wire-mesh floor and allowed to acclimatize for 15 min before being tested with von Frey filaments with logarithmically incremental stiffness (Chaplan et al., 1994). These filaments were applied to the lateral part of the plantar aspect of the hindpaw in the sural nerve territory (Decosterd and Woolf, 2000). A positive response was recorded when there was lifting or flinching of the stimulated paw. The $50 \%$ paw withdrawal threshold was calculated by Dixon's nonparametric test (Dixon, 1980; Chaplan et al., 1994). Kruskal-Wallis one-way ANOVA and Mann-Whitney post hoc test were used to determine whether there was a significant reduction in withdrawal threshold of the ipsilateral hindpaw in the SNI animals at each time point. $p<0.05$ was accepted as significant.

Tissue processing and immunocytochemistry. On the 7th or 28th postoperative day (see above), rats that had undergone SNI or sham operations were anesthetized deeply with pentobarbitone and perfused through the left ventricle with fixative containing $4 \%$ freshly depolymerized formaldehyde in $0.1 \mathrm{~m}$ phosphate buffer. An additional five male Sprague Dawley rats (two adult, 310 and $320 \mathrm{~g}$; three 14-d-old, $30 \mathrm{~g}$ ) were also anesthetized deeply and fixed by perfusion in the same way. Tissue from these animals was used for investigation of cleaved caspase- 3 immunoreactivity in naive adult spinal cord and for both caspase and TUNEL staining in the developing olfactory bulb. The L4 spinal cord segments from all adult rats were dissected out and stored for $8 \mathrm{~h}$ in the same fixative at $4^{\circ} \mathrm{C}$. The lengths of this segment in the rats that survived $28 \mathrm{~d}$ were measured so that we could determine whether there was any alteration after nerve injury. This was done by photographing the spinal cord in situ and measuring the distance between the points of entry of the most rostral and most caudal rootlets of the L4 dorsal root (Polgár et al., 2004). Spinal cord blocks from all operated animals were notched to allow the two sides to be distinguished, and transverse $60-\mu \mathrm{m}$-thick sections were cut with a Vibratome, rinsed in buffer, and subsequently treated with $50 \%$ ethanol to improve antibody penetration. Olfactory bulbs were removed from the 14-d-old animals and sectioned in the same way.

To investigate neuronal packing density in the dorsal horn after SNI, sections from the caudal half of the L4 spinal segment from operated animals that survived $28 \mathrm{~d}$ (SNI, $n=6$; sham operated, $n=6$ ) were incubated in monoclonal antibody against neuronal-specific nuclear protein (NeuN) (1:1000; Chemicon, Chandlers Ford, UK) and guinea pig antiserum against the neurokinin 1 (NK1) receptor (Polgár et al., 1999) (1:1000; this was used to define the lamina I/II border; see below), and then in the following secondary antibodies: anti-mouse IgG conjugated to cyanine 5.18 (Cy5) and anti-guinea pig IgG conjugated to Alexa 488. Sections were then stained with propidium iodide (1\% in PBS;
Sigma, Poole, UK) in the presence of RNase (10 mg/ml; Sigma) to reveal cell nuclei (Todd et al., 1998).

For all parts of the study, antibodies were made up in PBS containing $0.3 \mathrm{M} \mathrm{NaCl}$ and $0.3 \%$ Triton X-100. Species-specific secondary antibodies were raised in donkey and labeled with Alexa 488 (1:500; Molecular Probes, Eugene, OR) or rhodamine red or Cy5 (1:100; Jackson ImmunoResearch, West Grove, PA). Antibody incubations were at $4^{\circ} \mathrm{C}$ for $1-3$ $\mathrm{d}$ (primary antibodies) or for 2-16 h (secondary antibodies).

To look for evidence of cell death affecting neurons or other cell populations, sections from the L4 spinal segment of rats that survived $7 \mathrm{~d}$ after SNI and from the adult naive animals and sections of olfactory bulb from the 14-d-old rats were processed according to one or more of the following protocols: (1) incubation in rabbit antibody against cleaved caspase-3 (1:500; catalog number 9661; Cell Signaling Technology, Beverly, MA) and NeuN (as above) followed by secondary antibodies labeled with Alexa 488 or Cy5 and propidium iodide (as above); (2) sequential incubation in anti-cleaved caspase-3, Alexa 488-labeled donkey antirabbit IgG, rabbit anti-glial fibrillary acidic protein (GFAP; a marker for astrocytes; 1:2000; DakoCytomation, Ely, UK), Cy5-labeled donkey antirabbit IgG, and propidium iodide; (3) TUNEL staining (ApopTag Plus Fluorescein kit; catalog number S7111; according to the manufacturer's instructions; Chemicon) followed by incubation in anti-cleaved caspase- 3 and NeuN antibody, which were revealed with secondary antibodies labeled with rhodamine red and Cy5, and nuclear staining with 4',6-diamidino-2-phenylindole (DAPI); (4) TUNEL staining (as above) followed by incubation in monoclonal mouse antibody against adenomatous polyposis coli protein (APC; a marker for oligodendrocytes; clone CC1; 1:2000; Merck Biosciences, Nottingham, UK) and rabbit anti-GFAP (as above), which were revealed with rhodamine- and Cy5labeled secondary antibodies, and staining with DAPI; (5) TUNEL staining (as above) followed by incubation in rabbit antibody against ionized calcium-binding adapter molecule 1 (Iba-1; a marker for microglia; 1:1000; Wako Chemicals GmbH, Neuss, Germany), which was revealed with Cy5-labeled secondary antibody and staining with DAPI. Each of these protocols was performed on sections of $\mathrm{L} 4$ from all six rats that had undergone SNI $7 \mathrm{~d}$ previously, except for protocol 2, which was performed on sections from two of these animals. Sections from the L4 spinal segment of adult naive rats $(n=2)$ were processed according to protocols 1,2 , and 3 , whereas sections of olfactory bulb from the 14-dold rats $(n=3)$ were processed according to protocols 1 and 3 . Antibody against Iba-1 (a calcium-binding protein that is present in microglia but not neurons or astrocytes) (Imai et al., 1996; Ito et al., 1998; Svensson et al., 2005) was used instead of monoclonal antibody OX-42 to detect microglia, because staining with the latter antibody was found to be strongly suppressed by fixation for $>4 \mathrm{~h}$. In naive spinal cord sections that had been postfixed for up to $4 \mathrm{~h}$, complete colocalization of OX-42 and Iba-1 immunostaining was observed (data not shown).

Stereological analysis for neuronal counts. This was performed on sections from rats that had survived $28 \mathrm{~d}$ after SNI $(n=6)$ or sham operation $(n=6)$. From each animal, a single section from the caudal half of $\mathrm{L} 4$ was selected randomly and scanned with a confocal laser microscope (MRC 1024; Bio-Rad, Hemel Hempstead, UK) equipped with a krypton-argon laser. Sections were scanned sequentially with each laser line through a $60 \times$ oil-immersion lens to produce $z$-series of 16 optical sections separated by $1 \mu \mathrm{m}$ intervals, starting at least $5 \mu \mathrm{m}$ below the section surface. Because the field with this lens is a square of $\sim 150 \times 150 \mu \mathrm{m}$, for each Vibratome section, 18-20 slightly overlapping z-series were scanned to cover the whole of laminas I-III on each side. Lower-magnification images of each section were scanned through 4,10 , and $20 \times$ objective lenses with light transmitted through a dark-field condenser, and the sections were also scanned through the 10 and $20 \times$ objective lenses to reveal the NK1 receptor.

The stereological analysis was performed by an observer who was blind to both the treatment group from which the section was taken (SNI or sham operated) and the side (left or right). The analysis was performed as described previously (Polgár et al., 2004) by using a modification of the optical disector method (Williams and Rakic, 1988; Coggeshall, 1992; Bjugn and Gundersen, 1993; West, 1999; Guillery, 2002) to provide unbiased counts of neuronal nuclei in laminas I-III. Briefly, merged images 
representing NeuN and propidium iodide from the z-series obtained with the $60 \times$ objective were viewed with Neurolucida for Confocal (MicroBrightField, Colchester, VT). In each z-series, the third optical section was designated as the reference section and the 14th as the look-up section. Each optical section in the series was examined, and all neuronal nuclei (identified by the presence of both propidium iodide and NeuN) that were present in the reference section or appeared in subsequent sections in the series, but had disappeared by the look-up section, were drawn onto an outline of the gray matter. The borders between laminas I, II, and III were plotted from low-magnification scans of NK1 receptor immunoreactivity (laminas I-II) and those obtained through a darkfield condenser (laminas II-III) (Polgár et al., 2004). Because there is no obvious change in cytoarchitecture at the lamina III/IV border, its position was determined by reference to a standard atlas (Molander et al., 1984).

In the conventional disector method, separation between reference and look-up sections is set such that no structure can fall entirely between them (Sterio, 1984; Coggeshall, 1992). We were able to use a larger separation $(11 \mathrm{~mm})$ because all intervening sections were examined, and thus any neuronal nuclei that lay entirely between reference and look-up sections were included in the sample. By using a greater separation between reference and look-up sections, we were able to obtain a larger sample of neurons from each section. Because tissue shrinkage was found to occur in most sections (see Results), we used the following formula to estimate the number of neurons in each lamina on each side per $10 \mu \mathrm{m}$ length of spinal cord: $N=n \times T_{\text {final }} / T_{\text {cut }} \times 0.9091$, where $N$ is the number of neurons per $10 \mu \mathrm{m}$ of spinal cord, $n$ is the number counted in the $11 \mu \mathrm{m}$ disector, $T_{\text {final }}$ is the thickness of the particular section measured after any shrinkage, and $T_{\text {cut }}$ the original section thickness cut (60 $\mu \mathrm{m})$ (Polgár et al., 2004).

For both SNI and sham-operated rats, left (ipsilateral) and right (contralateral) sides of the spinal cord were analyzed separately, and results were compared with data from four naive rats (Sprague Dawley; 280-315 $\mathrm{g}$; right and left sides grouped together) that were included in the study by Polgár et al. (2004). Values were tested for statistical significance by one-way ANOVA.

Analysis of cleaved caspase-3 and TUNEL staining. Five sections from the $\mathrm{L} 4$ segment processed according to protocol 1 (see above) from each of the six rats that had undergone SNI $7 \mathrm{~d}$ previously were initially examined with a Bio-Rad Radiance 2100 confocal microscope. A search was made throughout the whole of laminas I-III in both dorsal horns in all sections to determine whether cells strongly or moderately labeled for cleaved caspase-3 (see Results) were NeuN immunoreactive. A detailed stereological analysis was also performed on two of these sections (selected randomly) from each animal. These were scanned through a $40 \times$ oil-immersion lens, and z-series of 21 optical sections at $1 \mu \mathrm{m}$ separation starting at the top surface of the section were obtained. The area covered with this lens was $300 \times 300 \mu \mathrm{m}$ and the region scanned included laminas I-III (and part of lamina IV) of the medial third of the dorsal horn on each side. Images were analyzed with Neurolucida for Confocal by an observer who was blind to which side was ipsilateral or contralateral to the SNI operation. All NeuN-positive cells for which the bottom surface of the nucleus was between the 5 th and 17 th optical section were selected, and the presence or absence of cleaved caspase-3-immunoreactivity was noted for each of these cells.

Sections processed according to protocol 2 (above) from two rats that had undergone SNI $7 \mathrm{~d}$ previously were analyzed to determine whether cleaved caspase-3 immunoreactivity was present in astrocytes. Two sections from each rat were scanned through their full depth with a $40 \times$ oil-immersion lens at $1 \mu \mathrm{m} \mathrm{z}$-spacing. Confocal image stacks showing cleaved caspase- 3 immunoreactivity were first viewed (by an observer blind to which side was ipsilateral or contralateral to the nerve injury), and all cells showing strong or moderate immunoreactivity were selected (between 167-203 per side for each rat). The image stacks corresponding to GFAP immunoreactivity were then examined and the presence or absence of GFAP-staining was noted for each of the selected cells. In a separate analysis, between 148 and 191 astrocytes were selected from each side in each animal, and the presence or absence of cleaved caspase- 3 in their nuclei was noted.

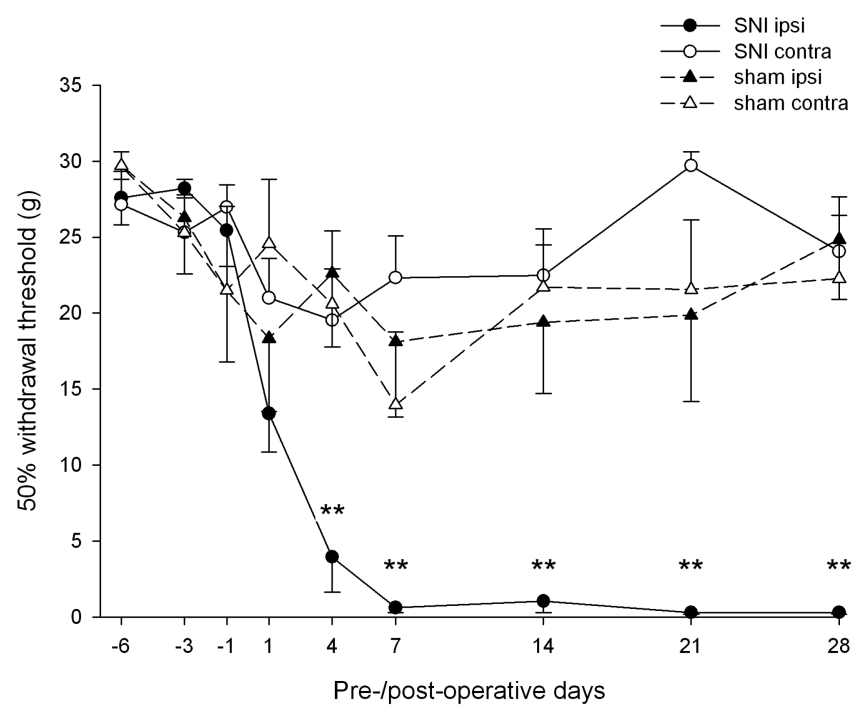

Figure 1. Graph showing $50 \%$ withdrawal thresholds to von Frey hairs in SNI ( $n=12 \leq 7 \mathrm{~d}$; $n=6 \geq 14 \mathrm{~d}$ ) and sham-operated (sham; $n=6$ ) rats. Results for ipsilateral (ipsi) and contralateral (contra) hindpaws are shown for each group, and each point represents the mean \pm SEM. **Significant difference $(p<0.01)$ between the ipsilateral paw in SNI animals and contralateral paw in these animals and both paws in the sham-operated group (Kruskal-Wallis one-way ANOVA with Mann-Whitney post hoc test).

Five sections reacted according to protocol 3 from the L4 segment of each rat that had undergone SNI $7 \mathrm{~d}$ previously were analyzed. Sections were scanned with a Radiance 2100 and the distribution of all TUNELpositive nuclei (defined by the presence of both TUNEL staining and DAPI) in the dorsal half of the spinal cord on both sides was plotted onto an outline of the gray and white matter. For each of these nuclei, the size was measured with Neurolucida for Confocal, and the presence or absence of immunoreactivity for NeuN and cleaved caspase- 3 was noted. Five sections processed by protocols 4 and 5 from each of these rats were also scanned and analyzed. All TUNEL-positive nuclei in the dorsal half of the ipsilateral side of the cord were examined to determine whether they showed immunoreactivity for GFAP or APC (protocol 4) or Iba-1 (protocol 5).

\section{Results}

\section{Behavior}

All of the rats that underwent the SNI procedure had an altered posture, holding the affected paw with the toes plantar-flexed and tending to avoid bearing weight on it. They all showed a substantial reduction in the $50 \%$ withdrawal threshold for the ipsilateral hindpaw, which developed during the first postoperative week (Fig. 1). The threshold values were significantly lower than those for the contralateral paw (and for both paws of the shamoperated animals) from day 4 to day 28 ( $p<0.01$; KruskalWallis test with Mann-Whitney post hoc test). By day 4, the threshold had dropped from a preoperative value of 25-28 to $4 \mathrm{~g}$; on days 7 and 14, it was at or below $1 \mathrm{~g}$; and at days 21 and 28, it was $0.3 \mathrm{~g}$. Sham-operated animals had normal posture and gait and did not show alterations in withdrawal threshold (Fig. 1).

\section{Analysis of neuronal density}

The distribution of NeuN immunoreactivity in sections from the caudal half of the L4 segment of both SNI and sham-operated animals that survived $28 \mathrm{~d}$ after operation was the same as that reported previously in naive rats (Todd et al., 1998; Polgár et al., 2004). No differences could be observed between the two sides of the SNI animals or between SNI and sham-operated rats.

The number of neurons that were included in the sample 


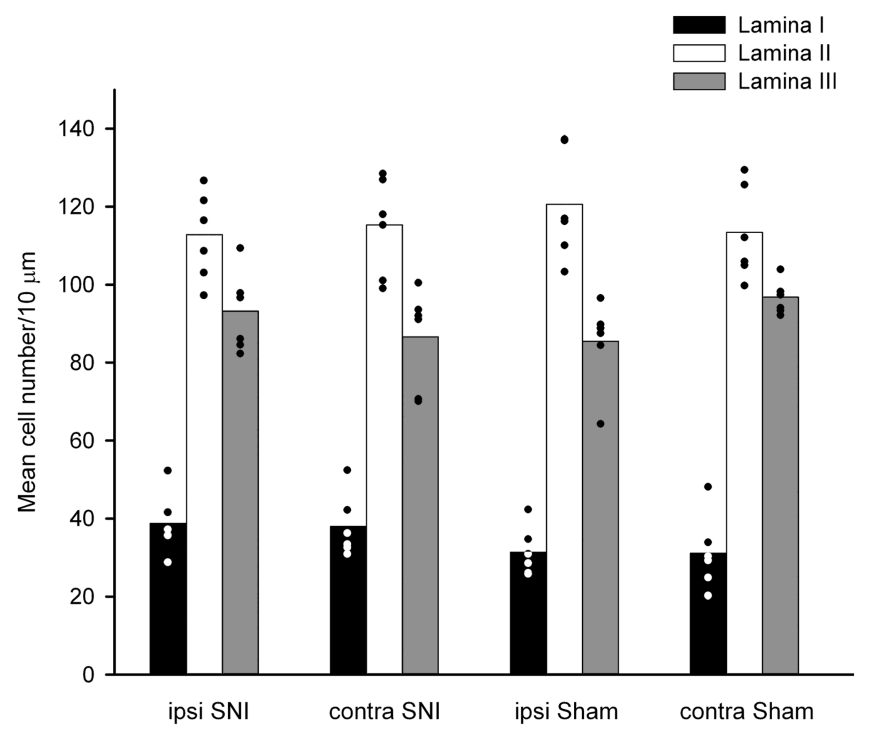

Figure 2. Graph showing the mean values for the estimated numbers of neurons per $10 \mu \mathrm{m}$ length of spinal cord for laminas I, II, and III in animals that had undergone SNI $(n=6)$ or sham operation ( $n=6) 28 \mathrm{~d}$ previously. The individual values from each animal are also indicated (circles). The numbers of NeuN-immunoreactive cells in each lamina on the side ipsilateral (ipsi) to the SNI operation did not differ significantly from those on the contralateral (contra) side or from the sham-operated rats (one-way ANOVA).

obtained with the disector method in each animal (SNI, $n=6$; sham operated, $n=6$ ) varied from 22 to 77 for lamina I, from 110 to 227 for lamina II, and from 85 to 172 for lamina III. Although the initial thickness of all sections was $60 \mu \mathrm{m}$, the thickness of the mounted sections, determined from scanning with the confocal microscope, varied from 40 to $60 \mu \mathrm{m}$ (Polgár et al., 2004). This indicates that shrinkage or compression had occurred in some of the sections during tissue processing. We therefore used a correction formula (see Materials and Methods) to estimate the number of neurons in each lamina on each side per $10 \mu \mathrm{m}$ length of spinal cord. These estimated numbers of neurons in laminas I, II, and III for the SNI and sham-operated rats are shown in Figure 2. The mean numbers of cells per $10 \mu \mathrm{m}$ length of spinal cord for laminas I, II, and III on the ipsilateral side of the SNI rats were $38.8 \pm 7.9( \pm \mathrm{SD}), 112.8 \pm 11.3$, and $93.2 \pm 10.5$, respectively. Corresponding values for the contralateral side of these rats were $38 \pm 8.2,115.3 \pm 12.6$, and $86.6 \pm 12.9$, whereas those for the sham-operated rats were $31.4 \pm 6.4,120.6 \pm 14.2$, and $85.5 \pm$ 11.1 on the ipsilateral side and $31.1 \pm 9.7,113.4 \pm 12.1$, and $96.8 \pm 4.3$ on the contralateral side. We previously obtained mean values of $30.6,112.1$, and 89.5 neurons per $10 \mu \mathrm{m}$ for laminas I, II, and III of naive rats (Polgár et al., 2004). The numbers of neurons per $10 \mu \mathrm{m}$ length of spinal cord in laminas I, II, and III on the ipsilateral side in the SNI animals did not differ significantly from the corresponding numbers on the contralateral side, nor from those on either side of the sham-operated animals, nor from those of the naive rats in the study by Polgár et al. (2004) (one-way ANOVA; $p=0.19$ for lamina I; $p=0.89$ for lamina II; $p=0.31$ for lamina III).

The mean length of the L4 segment in the SNI rats was $2.54 \pm$ $0.3 \mathrm{~mm}( \pm \mathrm{SD})$, whereas that for the sham-operated animals was $2.62 \pm 0.14 \mathrm{~mm}$. These values were not significantly different $(p=0.69 ;$ test $)$.

\section{Cleaved caspase- 3}

Many nuclei with a moderate or high level of cleaved caspase-3 immunoreactivity were seen throughout the gray matter of the
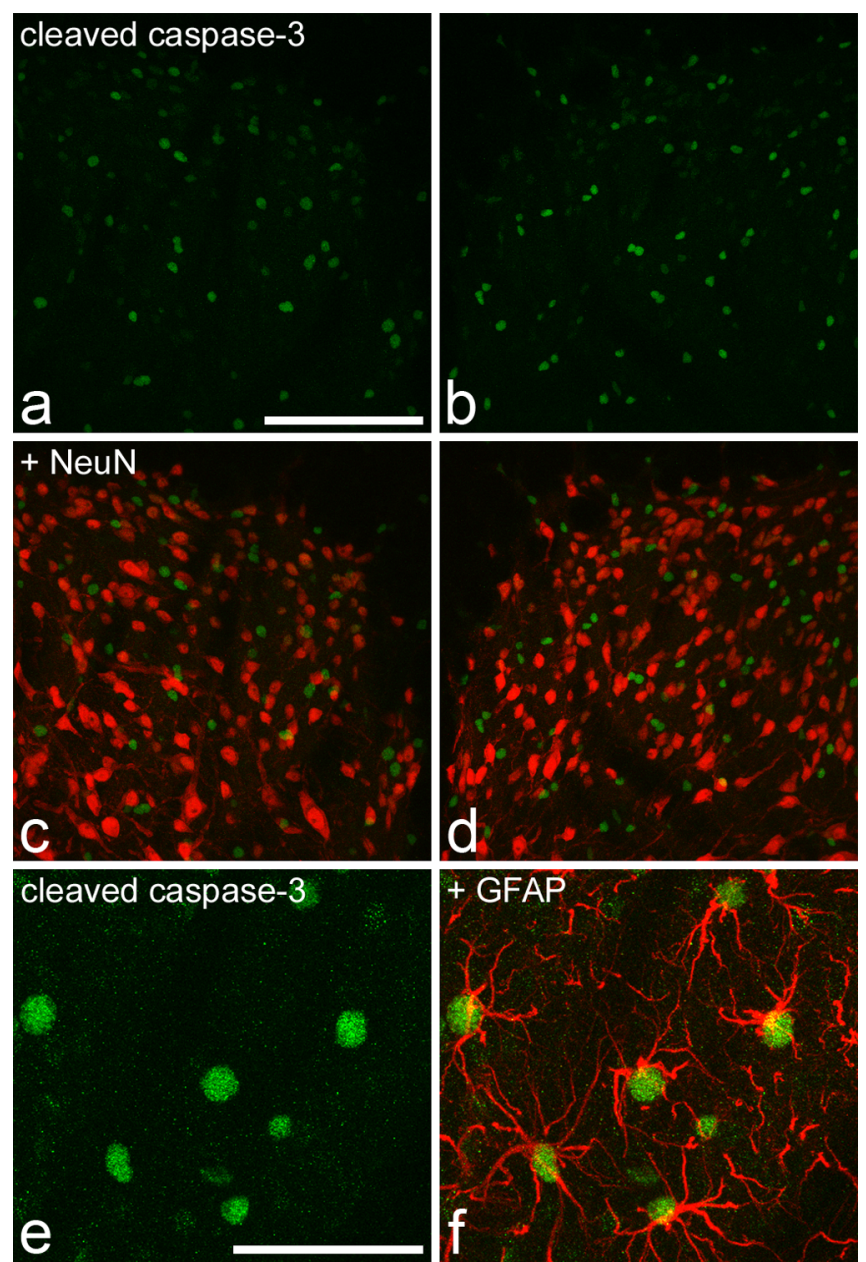

Figure 3. Cleaved caspase-3 immunoreactivity in the spinal cord of rats that had undergone SNI 7 d previously. $\boldsymbol{a}$ and $\boldsymbol{b}$ show staining for cleaved caspase-3 (green) in the medial part of the ipsilateral $(\boldsymbol{a})$ and contralateral $(\boldsymbol{b})$ dorsal horn. Numerous immunostained profiles are visible. $\boldsymbol{c}$, $\boldsymbol{d}$, Merging these images with NeuN staining (red) shows that the nuclei with strong or moderate immunoreactivity for cleaved caspase-3 are not NeuN positive. The rare instances of yellow in the merged images result from overlap of structures that are in different z-sections. $\boldsymbol{e}, \boldsymbol{f}$, Part of the ipsilateral dorsal horn from another rat that had undergone SNI. This section has been reacted sequentially with antibodies against cleaved caspase-3 (green) and GFAP (red). The nuclei with strong caspase immunoreactivity are surrounded by GFAP staining and can therefore be identified as astrocytes. Scale bars: (in $\boldsymbol{a}) \boldsymbol{a}-\boldsymbol{d}, 100 \mu \mathrm{m}$; (in $\boldsymbol{e}) \boldsymbol{e}, \boldsymbol{f}, 50 \mu \mathrm{m}$. Images are projected from $21(\boldsymbol{a}-\boldsymbol{d})$ or $15(\boldsymbol{e}, \boldsymbol{f}) \mathrm{z}$-sections at $1 \mu \mathrm{m}$ separation.

spinal cord on both sides in the six rats that had undergone SNI $7 \mathrm{~d}$ previously (Fig. 3a,b). The morphology of these nuclei, as revealed with propidium iodide, appeared normal. A careful search throughout laminas I-III of both dorsal horns in five sections from each animal revealed that none of these nuclei was NeuN positive (or surrounded by NeuN immunoreactivity) (Fig. $3 c, d)$. Very weak cleaved caspase-3 immunoreactivity was seen in a small number of NeuN-immunoreactive cells, which also had normal nuclear morphology. These were present in both ipsilateral and contralateral dorsal horns in each animal (data not shown) and were analyzed quantitatively. The proportion of NeuN-positive cells in the region scanned (medial third of laminas I-III/IV) that showed this weak cleaved caspase-3 immunoreactivity was $1.95 \pm 0.51 \%( \pm \mathrm{SEM})$ on the ipsilateral side and $1.70 \pm 0.24 \%$ on the contralateral side. These values were not significantly different ( $p=0.67 ; t$ test). To determine whether the cells with strong cleaved caspase- 3 immunoreactivity were astrocytes, we examined the relationship of this immunoreactiv- 


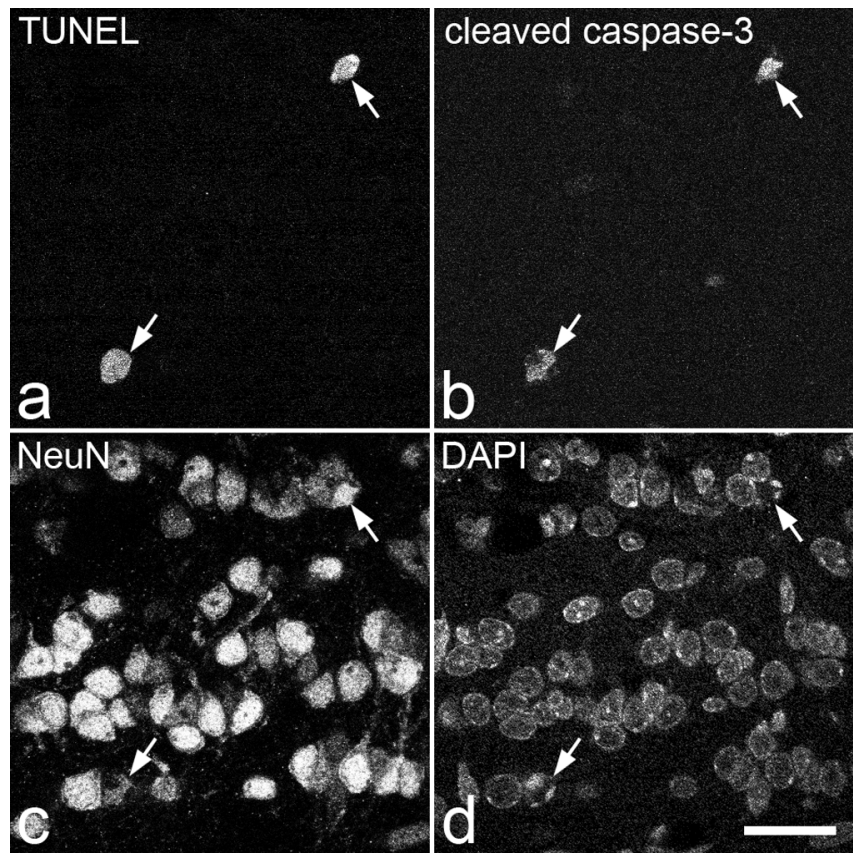

Figure 4. Cleaved caspase-3 and TUNEL staining in the olfactory bulb of a naive 14-d-old rat. $\boldsymbol{a}, \boldsymbol{b}$, A single optical section shows two nuclei (arrows) that are TUNEL stained and immunoreactive for cleaved caspase-3. c, Both nuclei are stained with the NeuN antibody.d, Staining with DAPI shows that both cells have abnormal nuclear morphology, with peripherally clumped chromatin. Scale bar, $20 \mu \mathrm{m}$.

ity to that obtained with antibody against GFAP in two of the SNI rats. The proportions of cells with strong cleaved caspase-3 immunoreactivity that were also GFAP positive were 88 and $92 \%$ in the ipsilateral dorsal horns and 87 and $91 \%$ in the contralateral dorsal horns. This indicates that the great majority of cells in the dorsal horn with high levels of cleaved caspase- 3 immunoreactivity were astrocytes (Fig. 3e,f). Between 98 and 100\% of the astrocytes (identified by the presence of GFAP immunostaining) sampled in these sections showed moderate or strong immunoreactivity for cleaved caspase-3. An identical staining pattern for cleaved caspase-3 was seen in the spinal cords from two naive adult rats, with moderate or strong staining in the nuclei of astrocytes and very faint staining in some neuronal nuclei (data not shown).

To confirm that cleaved caspase- 3 could be detected in degenerating neurons, we examined the olfactory bulb of 14-d-old rats, because neuronal apoptosis has been reported here at this stage of development (Najbauer and Leon, 1995). In each section, a few cells $(\sim 10-20$ per section) with strong staining for cleaved caspase- 3 were present in the anterior olfactory nucleus. Most of these cells showed abnormal nuclear morphology and many were also NeuN immunoreactive (Fig. 4).

\section{TUNEL staining}

Although the TUNEL reactions were performed on $60 \mu \mathrm{m} \mathrm{Vi-}$ bratome sections, TUNEL-positive nuclei (identified by the presence of both TUNEL staining and DAPI) were almost exclusively present near the section surfaces, presumably because of limited penetration of reagents into the tissue. TUNEL-positive nuclei were observed on the side ipsilateral to the nerve lesion in most sections from rats that had undergone SNI $7 \mathrm{~d}$ previously (7-22 nuclei per five sections from each rat; mean, $14.3 \pm 6.1 \mathrm{SD} ; n=6$ ) but were seldom seen on the contralateral side (one to four nuclei per five sections; mean, $2.2 \pm 1.5)$. This difference was statistically significant $(p<0.005 ; t$ test). They were seldom seen in the
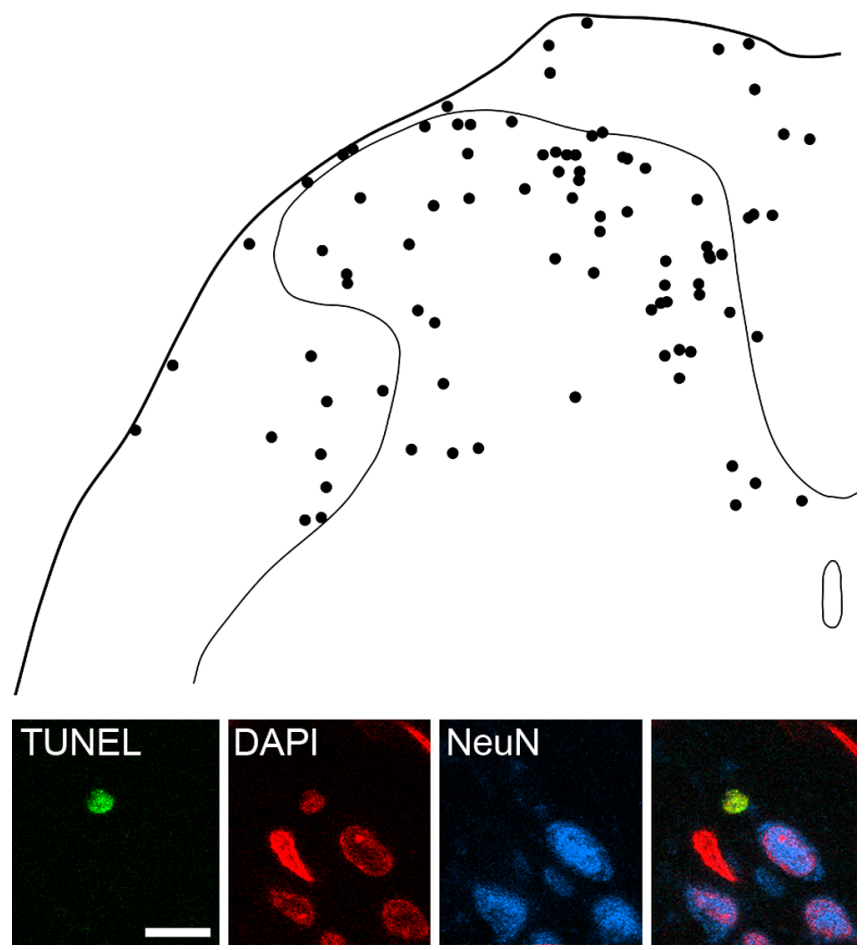

Figure 5. TUNEL staining in the dorsal spinal cord $7 \mathrm{~d}$ after SNI and lack of coexistence with NeuN immunoreactivity. The drawing shows the positions of 86 TUNEL-positive nuclei that were present on the side ipsilateral to the SNI in five sections each from six rats, plotted onto an outline of the dorsal half of the spinal cord. Note that although the majority of TUNEL-positive nuclei are in the gray matter, there are also many throughout the white matter. The confocal images in the bottom part of the figure show a single optical section through a TUNEL-labeled nucleus in lamina I that is not NeuN immunoreactive. Other NeuN-positive and NeuN-negative nuclei are also visible. Scale bar for confocal images, $10 \mu \mathrm{m}$.

spinal cords of naive adult rats (zero to two nuclei per five sections from each rat; $n=2$ ). TUNEL-positive nuclei were small, generally had a pyknotic appearance, and measured between 3.0 and 7.2 $\mu \mathrm{m}$ (mean, $5.1 \pm 0.9 \mathrm{SD}$ ) in their maximum dimension and between 2.6 and $5.9 \mu \mathrm{m}$ (mean, $3.9 \pm 0.7$ ) in the minimum dimension. They were distributed widely throughout both gray and white matter of the dorsal half of the spinal cord on the ipsilateral side (Fig. 5), and there were no significant differences in size between those in the gray matter $(4.99 \pm 0.8 \times 3.8 \pm 0.6$ $\mu \mathrm{m} ; n=59)$ and those in the white matter $(5.27 \pm 1.1 \times 4.0 \pm 0.7$ $\mu \mathrm{m} ; n=27$ ) ( $t$ test; $p>0.1)$. They were present in all dorsal horn laminas and in the white matter of the dorsal columns, dorsal root entry zone, lateral white matter, and Lissauer's tract. In sections reacted according to protocol 3, none of the 86 TUNELpositive nuclei on the ipsilateral side were immunoreactive for NeuN (Fig. 5). TUNEL-positive nuclei were sometimes located in close proximity to neurons, and in a few cases, there was overlap of fluorescence for the TUNEL stain and NeuN in individual optical sections (Fig. 6, insets). However, it was always possible to show that these neurons had intact nuclei with normal morphology that were quite distinct from the adjacent TUNEL-positive nuclei (Fig. 6). Cleaved caspase-3 immunoreactivity was detected in 14 of the 86 TUNEL-positive nuclei analyzed in these sections (data not shown): six of these were in laminas I-III, and the remainder were in the white matter.

In sections of olfactory bulb from 14-d-old rats that were processed by protocol 3, some nuclei with cleaved caspase- 3 immunoreactivity were TUNEL positive and others were TUNEL negative. NeuN immunoreactivity was found in some of the nu- 


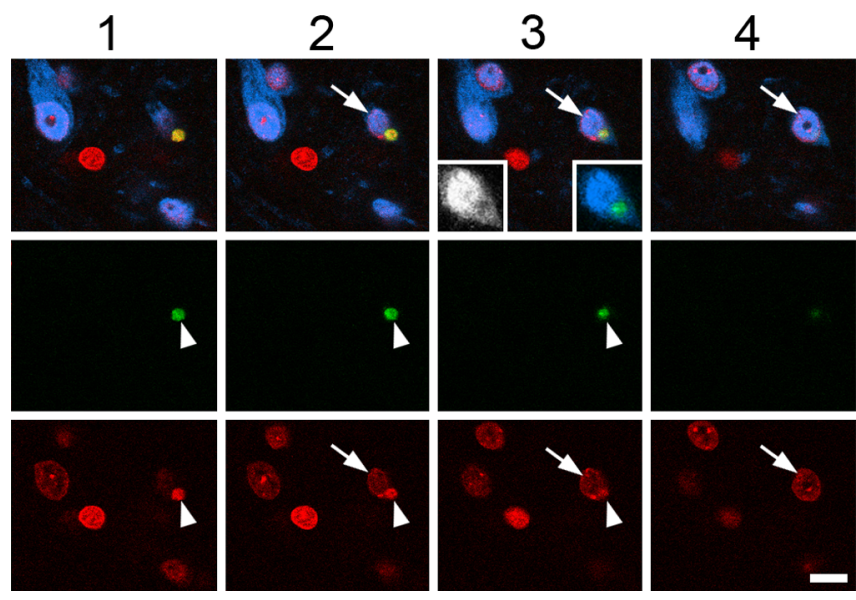

Figure 6. The appearance of TUNEL staining, NeuN immunoreactivity, and DAPI in four consecutive optical sections (1-4) through the ipsilateral dorsal horn of a rat that underwent SNI 7 d previously. For each optical section, the top panel shows a merged image (NeuN, blue; TUNEL, green; DAPI, red), the middle panel shows TUNEL staining (green), and the bottom panel shows DAPI staining (red). A small TUNEL-positive nucleus with highly condensed chromatin is visible in the first three sections (arrowheads) and is closely apposed to a NeuNimmunoreactive cell with normal nuclear morphology (arrows), which can be seen in sections 2-4. Although the sequence of images scanned for DAPI clearly shows that these are two separate cells, the TUNEL labeling overlaps with NeuN immunoreactivity in the third section (shown with and without TUNEL labeling in insets) and could easily be mistaken for a TUNELpositive NeuN-immunoreactive cell. z-spacing between sections, $1 \mu \mathrm{m}$; scale bar, $10 \mu \mathrm{m}$.

clei in both of these groups (Fig. 4). In addition, there were nuclei that lacked cleaved caspase- 3 immunoreactivity but were moderately or strongly stained with the TUNEL method. NeuN immunoreactivity was found in some of these nuclei but was absent from those with the most intense TUNEL staining.

Ninety-four TUNEL-positive nuclei were identified in the ipsilateral dorsal spinal cord in sections reacted according to protocol 4 taken from the six rats that had undergone SNI $7 \mathrm{~d}$ previously (10-21 per rat). APC immunoreactivity was seen in the perikaryal cytoplasm and proximal processes of many cells, which were presumably oligodendrocytes. However, none of these cells, and none of the GFAP-immunoreactive astrocytes, had TUNEL-positive nuclei. In sections reacted according to protocol 5, Iba-1-immunoreactive microglia were present throughout the gray and white matter of the spinal cord, and these appeared to be more common on the ipsilateral side. Iba-1 immunoreactivity was present in the perikaryal cytoplasm (and to a lesser extent in the nucleus) of these cells and extended into their processes. Ninety-three TUNEL-positive nuclei were observed in the ipsilateral dorsal spinal cord in these sections (12-21 per rat). The great majority of these $(87,94 \%)$ were surrounded by Iba-1 immunoreactivity and could clearly be identified as microglia (Fig. 7). In the remaining cases, the TUNEL-positive nuclei were associated with disrupted patches of Iba-1 immunoreactivity, and these may represent microglial cells that were at a later stage of degeneration.

\section{Discussion}

The major findings of this study were: (1) that there was no detectable loss of neurons from laminas I-III of the ipsilateral dorsal horn 4 weeks after operation in the SNI model and (2) that neither cleaved caspase-3 nor TUNEL staining provided evidence of degenerating neurons in the dorsal horn 1 week after SNI. Although TUNEL-positive (presumed apoptotic) cells were present throughout the white and gray matter of the dorsal spinal
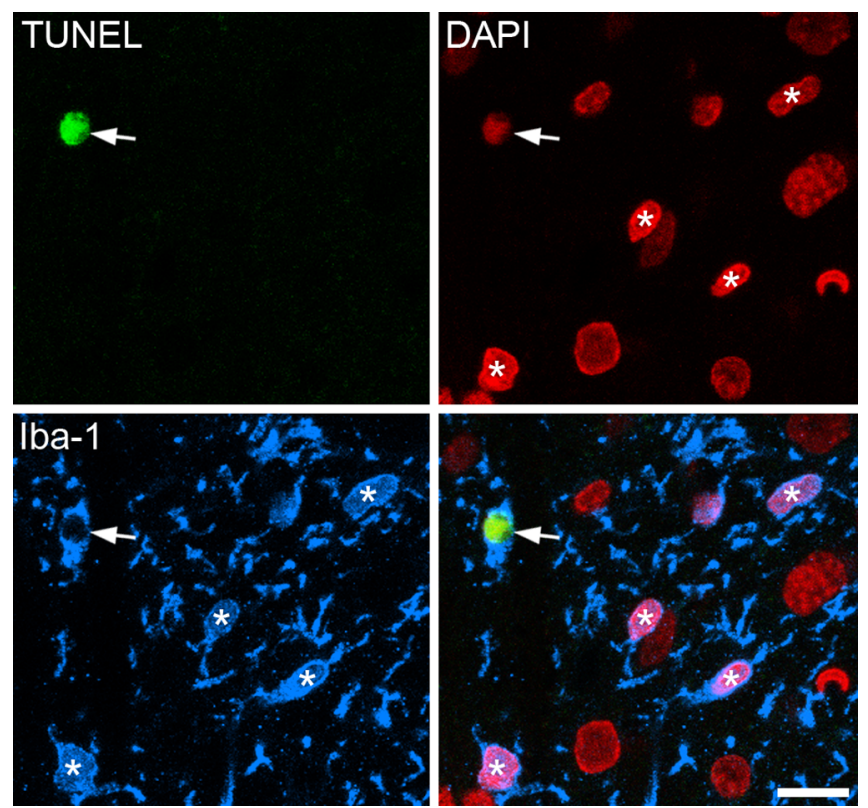

Figure 7. TUNEL staining in a microglial cell in the superficial dorsal horn from a rat that had undergone SNI $7 \mathrm{~d}$ previously. The four panels show staining for TUNEL (green), DAPI (red), and Iba-1 (blue), together with a merged image. The nucleus of an Iba-1-immunoreactive microglial cell shows TUNEL staining (arrows). Several other microglia with TUNEL-negative nuclei are also visible, and some of these are marked with asterisks. Projected from three optical sections at $1 \mu \mathrm{m} z$-spacing. Scale bar, $10 \mu \mathrm{m}$.

cord on the ipsilateral side 1 week after operation, they expressed Iba- 1 and were therefore presumably microglia.

\section{Numbers of neurons in laminas I-III}

The hypothesis that significant numbers of dorsal horn neurons die after nerve injury is based on two main types of evidence: counts of the numbers of neurons in a specified volume of the dorsal horn (packing density) and use of the TUNEL method to identify cells that are undergoing apoptosis. de Novellis et al. (2004) reported an apparent reduction of $\sim 50 \%$ in the number of neurons in lamina II on both sides of the spinal cord $7 \mathrm{~d}$ after CCI. However, these authors previously detected a reduction of only $23 \%$ of neurons in laminas I-III on the ipsilateral side $14 \mathrm{~d}$ after CCI (Maione et al., 2002). It is hard to reconcile these two findings, and because they were both obtained from simple profile counts, rather than with a stereological method (see below), they must be treated with considerable caution. Two studies that have used stereology have provided evidence for loss of dorsal horn neurons after nerve injury. Coggeshall et al. (2001) found a reduction in neuronal numbers in laminas I-III of the ipsilateral dorsal horn after sciatic nerve transection but only when this was followed by electrical stimulation of the severed nerve at a stimulus strength sufficient to activate $\mathrm{A} \beta$ afferents; there is also a preliminary account of neuronal loss from these laminas in three neuropathic models: SNI, CCI, and spinal nerve ligation (Scholz et al., 2003). In contrast, we have reported that there is no reduction in the packing density of neurons in laminas I, II, or III on the ipsilateral side of the spinal cord $14 \mathrm{~d}$ postoperatively in rats that had undergone CCI, despite the presence of thermal hyperalgesia in these animals (Polgár et al., 2004).

It is well established that unbiased stereological methods are needed to provide reliable cell counts (Coggeshall, 1992; Bjugn and Gundersen, 1993; West, 1999; Guillery, 2002), and we therefore used a modification of the disector method (Polgár et al., 
2004) in this study. We immunostained sections with the NeuN antibody (Mullen et al., 1992) because this appears to label all neurons in the spinal cord but not glial cells (Todd et al., 1998). Although NeuN immunostaining is concentrated in neuronal nuclei, there also is labeling of perikaryal cytoplasm in some cells, and this can extend into proximal dendrites. This variation in NeuN labeling between neurons means that it is difficult to distinguish the extent of individual cells with NeuN alone, and for this reason, we used the $\mathrm{z}$-positions of the bottom surfaces of their nuclei (stained with propidium iodide) as the inclusion/ exclusion criterion for the disector method. We examined the entire cross-sectional area of laminas I-III, because TUNELpositive cells have been found throughout these laminas (Azkue et al., 1998). Scholz et al. (2003) reported that apoptosis in neuropathic models can persist for longer than 3 weeks, and we therefore analyzed neuronal packing density at a 4 week survival time, when any neuronal loss would presumably be maximal. Our finding that the numbers of neurons per $10 \mu \mathrm{m}$ in laminas I-III were not reduced on the ipsilateral side in rats that had undergone SNI 4 weeks previously strongly suggests that there was not a significant degree of neuronal death in these laminas in our SNI animals.

\section{Detection of apoptotic cells with antibody against cleaved caspase- 3 and TUNEL staining}

We used two methods that detect different stages of the apoptosis cascade to look for evidence of dying neurons in the dorsal horn after SNI. Activation (cleavage) of caspase-3 precedes DNA damage and should therefore be detected in apoptotic cells at an earlier stage than TUNEL positivity. However, the two markers have an overlapping time course, as shown by their coexistence in some cells (Davoli et al., 2002) (Fig. 4). Both cleaved caspase-3 and TUNEL staining have been found to colocalize with NeuN in other pathological states (Katchanov et al., 2001; Araki et al., 2002; Davoli et al., 2002; Mao et al., 2002; Zhang et al., 2002; Korhonen et al., 2003; Lei et al., 2004) and can therefore be used to reveal apoptotic neurons. Our finding that each of these markers could be colocalized with $\mathrm{NeuN}$ in developing olfactory bulb (Fig. 4) demonstrates that it was possible to detect apoptotic neurons with our staining techniques.

Although it has been suggested that the presence of cleaved caspase- 3 represents a point of no return in the apoptotic pathway (Huppertz et al., 1999), this form of the enzyme has also been identified in cells that are not undergoing apoptosis (Oomman et al., 2004). We found that astrocytes throughout the spinal cord of both SNI and naive rats showed moderate to high levels of cleaved caspase-3, and a similar observation has been made in naive rat spinal cord in two previous studies (Martin and Liu, 2002; Hoang et al., 2003). Because virtually all astrocytes were immunoreactive with the cleaved caspase- 3 antibody, it is highly unlikely that this represents apoptosis in these cells. We found that only a very few neurons had detectable cleaved caspase-3, that this was invariably at very low levels, and that the pattern and frequency of expression in neurons did not differ between the two sides in the SNI rats. This, together with the complete lack of colocalization between NeuN immunoreactivity and TUNEL staining, suggests that neurons do not undergo apoptosis in this model.

TUNEL-positive cells have been seen in the dorsal horn after various types of nerve injury, including sciatic nerve transection and both the CCI and SNI models (Gehrmann and Banati, 1995; Kawamura et al., 1997; Azkue et al., 1998; Whiteside and Munglani, 2001; Moore et al., 2002; Scholz et al., 2003; de Novel- lis et al., 2004). Azkue et al. (1998), Whiteside and Munglani (2001), and Moore et al. (2002) reported similar numbers of TUNEL cells ( 1.6 per $25 \mu \mathrm{m}$ section, 1.5 per $15 \mu \mathrm{m}$ section, and 1.48 per $10 \mu \mathrm{m}$ section, respectively) after different types of nerve injury. We found on average 3.03 cells per $60 \mu \mathrm{m}$ section in the sections reacted by protocols 3,4 , and 5 , but because these sections were reacted free-floating with both surfaces exposed to reagents, and because TUNEL-positive cells were only present near the section surfaces, the numbers of TUNEL cells that we saw are consistent with those reported in these three studies.

Azkue et al. (1998) concluded that virtually all of the TUNELpositive cells that they saw after sciatic nerve transection were neurons, based on staining with antibody against neurofilaments. However, Gehrmann and Banati (1995) had shown previously that TUNEL labeling in the spinal cord after sciatic transection was present in microglia (identified by the binding of Bandeiraea simplicifolia isolectin B4), and Coggeshall et al. (2001) found no loss of neurons from laminas I-III in this model. It is therefore unlikely that significant neuronal apoptosis occurs after complete transection of the sciatic nerve. Moore et al. (2002) reported that $\sim 10 \%$ of TUNEL-positive nuclei seen after SNI were NeuN immunoreactive; however, our finding that TUNEL-positive nuclei were often in close proximity to neurons with intact nuclei (Fig. 6) suggests that they may have misinterpreted overlap between NeuN and TUNEL staining in adjacent cells. Interestingly, activated microglia have been shown to migrate along neurons after peripheral nerve injury, and this phenomenon is thought to underlie synaptic stripping (Schiefer et al., 1999). In addition, Gehrmann and Banati (1995) described apoptotic microglia in close proximity to neuronal cell bodies in the ventral horn after sciatic nerve transection.

The small size of the TUNEL-positive nuclei that we observed $(5.1 \times 3.9 \mu \mathrm{m})$ matches that reported for microglia in young adult brain (mean diameter, $4.1 \mu \mathrm{m}$ ) (Ling et al., 1973). This, together with their distribution in both gray and white matter, the fact that the great majority were Iba- 1 immunoreactive (but none were labeled for NeuN, APC, or GFAP), and their occasional association with neurons, strongly suggests that the apoptotic cells are all microglia, as has been reported after complete transection of the sciatic nerve (Gehrmann and Banati, 1995). Microglia are known to proliferate in the spinal cord after peripheral nerve injury (Gehrmann et al., 1991; Eriksson et al., 1993), and apoptosis is thought to be a homeostatic mechanism to reduce their number after activation (Gehrmann and Banati, 1995).

Although we cannot rule out the possibility that a small number of dorsal horn neurons die after peripheral nerve injury, our findings demonstrate that significant loss of neurons is not required for the development of tactile allodynia in the SNI model.

\section{References}

Araki T, Simon RP, Taki W, Lan JQ, Henshall DC (2002) Characterization of neuronal death induced by focally evoked limbic seizures in the C57BL/6 mouse. J Neurosci Res 69:614-621.

Azkue JJ, Zimmermann M, Hsieh T-F, Herdegen T (1998) Peripheral nerve insult induces NMDA receptor-mediated, delayed degeneration in spinal neurons. Eur J Neurosci 10:2204-2206.

Bennett GJ, Xie YK (1988) A peripheral mononeuropathy in rat that produces disorders of pain sensation like those seen in man. Pain 33:87-107.

Bjugn R, Gundersen HJ (1993) Estimate of the total number of neurons and glial and endothelial cells in the rat spinal cord by means of the optical disector. J Comp Neurol 328:406-414. 
Campbell JN, Raja SN, Meyer RA, Mackinnon SE (1988) Myelinated afferents signal the hyperalgesia associated with nerve injury. Pain 32:89-94.

Castro-Lopes JM, Tavares I, Coimbra A (1993) GABA decreases in the spinal cord dorsal horn after peripheral neurectomy. Brain Res 620:287-291.

Chaplan SR, Bach FW, Pogrel JW, Chung JM, Yaksh TL (1994) Quantitative assessment of tactile allodynia in the rat paw. J Neurosci Methods 53:55-63.

Coggeshall RE (1992) A consideration of neural counting methods. Trends Neurosci 15:9-13.

Coggeshall RE, Lekan HA, White FA, WoolfCJ (2001) A-fiber sensory input induces neuronal cell death in the dorsal horn of the adult rat spinal cord. J Comp Neurol 435:276-282.

Davoli MA, Fourtounis J, Tam J, Xanthoudakis S, Nicholson D, Robertson GS, Ng GY, Xu D (2002) Immunohistochemical and biochemical assessment of caspase- 3 activation and DNA fragmentation following transient focal ischemia in the rat. Neuroscience 115:125-136.

Decosterd I, Woolf CJ (2000) Spared nerve injury: an animal model of persistent peripheral neuropathic pain. Pain 87:149-158.

de Novellis V, Siniscalo D, Galderisi U, Fuccio R, Nolano M, Santoro L, Cascino A, Roth KA, Rossi N, Maione S (2004) Blockade of glutamate mGlu5 receptors in a rat model of neuropathic pain prevents early overexpression of pro-apoptotic genes and morphological changes in dorsal horn lamina II. Neuropharmacology 46:468-479.

Dixon WJ (1980) Efficient analysis of experimental observations. Annu Rev Pharmacol Toxicol 20:441-462.

Eaton MJ, Plunkett JA, Karmally S, Martinez MA, Montanez K (1998) Changes in GAD- and GABA-immunoreactivity in the spinal dorsal horn after peripheral nerve injury and promotion of recovery by lumbar transplants of immortalized serotoninergic precursors. J Chem Neuroanat 16:57-72.

Eriksson NP, Persson JK, Svensson M, Arvidsson J, Molander C, Aldskogius H (1993) A quantitative analysis of microglial cell reaction in central primary sensory projection territories following peripheral nerve injury in the adult rat. Exp Brain Res 96:19-27.

Gehrmann J, Banati R (1995) Microglial turnover in the injured CNS: activated microglia undergo delayed DNA fragmentation following peripheral nerve injury. J Neuropathol Exp Neurol 54:680-688.

Gehrmann J, Monaco S, Kreutzberg GW (1991) Spinal cord microglial cells and DRG satellite cells rapidly respond to transection of the rat sciatic nerve. Restor Neurol Neurosci 2:181-198.

Guillery RW (2002) On counting and counting errors. J Comp Neurol 447:1-7.

Hoang TX, Nieto JH, Tillakaratne NJ, Havton LA (2003) Autonomic and motor neuron death is progressive and parallel in a lumbosacral ventral root avulsion model of cauda equina injury. J Comp Neurol 467:477-486.

Huppertz B, Frank H-G, Kaufmann (1999) The apoptosis cascade-morphological and immunohistochemical methods for its visualization. Anat Embryol 200:1-18.

Ibuki T, Hama AT, Wang X-T, Pappas GD, Sagen J (1997) Loss of GABAimmunoreactivity in the spinal dorsal horn of rats with peripheral nerve injury and promotion of recovery by adrenal medullary grafts. Neuroscience 76:845-858.

Imai Y, Ibata I, Ito D, Ohsawa K, Kohsaka S (1996) A novel gene ibal in the major histocompatibility complex class III region encoding an EF hand protein expressed in a monocytic lineage. Biochem Biophys Res Commun 224:855-862.

Ito D, Imai Y, Ohsawa K, Nakajima K, Fukuuchi Y, Kohsaka S (1998) Microglia-specific localisation of a novel calcium binding protein, Ibal. Mol Brain Res 57:1-9.

Katchanov J, Harms C, Gertz K, Hauck L, Waeber C, Hirt L, Priller J, von Harsdorf R, Bruck W, Hortnagl H, Dirnagl U, Bhide PG, Endres M (2001) Mild cerebral ischemia induces loss of cyclin-dependent kinase inhibitors and activation of cell cycle machinery before delayed neuronal cell death. J Neurosci 21:5045-5053.

Kawamura T, Akira T, Watanabe M, Kagitani Y (1997) Prostaglandin E1 prevents apoptotic cell death in superficial dorsal horn of rat spinal cord. Neuropharmacology 36:1023-1030.
Koltzenburg M, Torebjork HE, Wahren LK (1994) Nociceptor modulated central sensitization causes mechanical hyperalgesia in acute chemogenic and chronic neuropathic pain. Brain 117:579-591.

Korhonen L, Belluardo N, Mudo G, Lindholm D (2003) Increase in Bcl-2 phosphorylation and reduced levels of BH3-only Bcl-2 family proteins in kainic acid-mediated neuronal death in the rat brain. Eur J Neurosci 18:1121-1134.

Lei B, Popp S, Capuano-Waters C, Cottrell JE, Kass IS (2004) Lidocaine attenuates apoptosis in the ischemic penumbra and reduces infarct size after transient focal cerebral ischemia in rats. Neuroscience 125:691-701.

Ling EA, Paterson JA, Privat A, Mori S, Leblond CP (1973) Investigation of glial cells in semithin sections. I. Identification of glial cells in the brain of young rats. J Comp Neurol 149:43-72.

Maione S, Siniscalo D, Galderisi U, de Novellis V, Uliano R, Di Bernardo G, Berrino L, Cascino A, Rossi N (2002) Apoptotic gene expression in the lumbar dorsal horn in a model neuropathic pain in rat. NeuroReport 13:101-106.

Mao J, Sung B, Ji RR, Lim G (2002) Neuronal apoptosis associated with morphine tolerance: evidence for an opioid-induced neurotoxic mechanism. J Neurosci 22:7650-7661.

Martin LJ, Liu Z (2002) Injury-induced spinal motor neuron apoptosis is preceded by DNA single-strand breaks and is p53- and Bax-dependent. J Neurobiol 50:181-197.

Molander C, Xu Q, Grant G (1984) The cytoarchitectonic organization of the spinal cord in the rat. I. The lower thoracic and lumbosacral cord. J Comp Neurol 230:133-141.

Moore KA, Kohno T, Karchewski LA, Scholz J, Baba H, Woolf CJ (2002) Partial peripheral nerve injury promotes a selective loss of GABAergic inhibition in the superficial dorsal horn of the spinal cord. J Neurosci 22:6724-6731.

Mullen RJ, Buck CR, Smith AM (1992) NeuN, a neuronal specific nuclear protein in vertebrates. Development 116:201-211.

Najbauer J, Leon M (1995) Olfactory experience modulates apoptosis in the developing olfactory bulb. Brain Res 674:245-251.

Oomman S, Finckbone V, Dertien J, Attridge J, Henne W, Medina M, Mansouri B, Singh H, Strahlendorf H, Strahlendorf J (2004) Active caspase-3 expression during postnatal development of rat cerebellum is not systematically or consistently associated with apoptosis. J Comp Neurol 476:154-173.

Polgár E, Shehab SAS, Watt C, Todd AJ (1999) GABAergic neurons that contain neuropeptide $\mathrm{Y}$ selectively target cells with the neurokinin 1 receptor in laminae III and IV of the rat spinal cord. J Neurosci 19:2637-2646.

Polgár E, Hughes DI, Riddell JS, Maxwell DJ, Puskár Z, Todd AJ (2003) Selective loss of spinal GABAergic or glycinergic neurons is not necessary for development of thermal hyperalgesia in the chronic constriction injury model of neuropathic pain. Pain 104:229-239.

Polgár E, Gray S, Riddell JS, Todd AJ (2004) Lack of evidence for significant neuronal loss in laminae I-III of the spinal dorsal horn of the rat in the chronic constriction injury model. Pain 111:144-150.

Schiefer J, Kampe K, Dodt HU, Zieglgänsberger W, Kreutzberg GW (1999) Microglial motility in the rat facial nucleus following peripheral axotomy. J Neurocytol 28:439-453.

Scholz J, Broom DC, Youn D, Kohno T, Mills CD, Suter M, Decosterd I, Lekan H, Coggeshall RE, Woolf CJ (2003) Neurodegeneration in the dorsal horn of the spinal cord contributes to persistent pain after peripheral nerve injury. Soc Neurosci Abstr 29:696.3.

Sivilotti L, Woolf CJ (1994) The contribution of GABAA and glycine receptors to central sensitization: disinhibition and touch-evoked allodynia in the spinal cord. J Neurophysiol 72:169-179.

Sterio DC (1984) The unbiased estimation of number and sizes of arbitrary particles using the disector. J Microsc 134:127-136.

Sugimoto T, Bennett GJ, Kajander KC (1990) Transsynaptic degeneration in the superficial dorsal horn after sciatic nerve injury: effects of a chronic constriction injury, transection, and strychnine. Pain 42:205-213.

Svensson CI, Fitzsimmons B, Azizi S, Powell HC, Hua XY, Yaksh TL (2005) Spinal p38beta isoform mediates tissue injury-induced hyperalgesia and spinal sensitization. J Neurochem 92:1508-1520. 
Todd AJ, Spike RC, Polgár E (1998) A quantitative study of neurons which express neurokinin 1 or somatostatin $s_{2} t_{2 a}$ receptor in rat spinal dorsal horn. Neuroscience 85:459-473.

Troy CM, Salvesen GS (2002) Caspases on the brain. J Neurosci Res 69:145-150.

West MJ (1999) Stereological methods for estimating the total number of neurons and synapses: issues of precision and bias. Trends Neurosci 22:51-61.

Whiteside GT, Munglani R (2001) Cell death in the superficial dorsal horn in a model of neuropathic pain. J Neurosci Res 64:168-173.

Williams RW, Rakic P (1988) Three-dimensional counting: an accurate and direct method to estimate numbers of cells in sectioned material. J Comp Neurol 278:344-352.
Woolf CJ (1997) Molecular signals responsible for the reorganization of the synaptic circuitry of the dorsal horn after peripheral nerve injury: the mechanisms of tactile allodynia. In: Molecular neurobiology of pain (Borsook D, ed), pp 171-200. Seattle: IASP.

Yaksh TL (1989) Behavioral and anatomic correlates of the tactileevoked allodynia produced by spinal glycine inhibition: effects of modulatory receptor systems and excitatory amino acid antagonists. Pain 37:111-123.

Zhang C, Siman R, Xu YA, Mills AM, Frederick JR, Neumar RW (2002) Comparison of calpain and caspase activities in the adult rat brain after transient forebrain ischemia. Neurobiol Dis 10:289-305.

Zimmerman M (2001) Pathobiology of neuropathic pain. Eur J Pharmacol 429:23-37. 